\title{
Ortho-Planar Linear-Motion Springs
}

John J. Parise

Larry L. Howell

lhowell@byu.edu

Spencer P. Magleby

magleby@byu.edu

Follow this and additional works at: https://scholarsarchive.byu.edu/facpub

Part of the Mechanical Engineering Commons

\section{Original Publication Citation}

Parise, J.J., Howell, L.L., and Magleby, S.P., â€œOrtho-Planar Linear-Motion Springs,â€

Mechanism and Machine Theory, Vol. 36, Nos. 11-12, pp. 1281-13, 21.

\section{BYU ScholarsArchive Citation}

Parise, John J.; Howell, Larry L.; and Magleby, Spencer P., "Ortho-Planar Linear-Motion Springs" (2001). Faculty Publications. 558.

https://scholarsarchive.byu.edu/facpub/558 


\title{
Ortho-Planar Linear-Motion Springs
}

by

\author{
John J. Parise, Larry L. Howell ${ }^{1}$, and Spencer P. Magleby \\ Mechanical Engineering Department \\ Brigham Young University \\ Provo, Utah 84602 \\ U.S.A. \\ Phone: 801-378-2625 \\ Fax: 801-378-5037 \\ Email: lhowell@et.byu.edu
}

\begin{abstract}
This paper presents an ortho-planar spring design that operates by raising or lowering its platform relative to the base with no rotation. The compact nature of the design, and its non-rotating motion, eliminates the problem of rotation against adjoining surfaces and is less sensitive to variation in assemblies than many current compact springs. Nomenclature is presented to identify different configurations, mathematical equations are provided that accurately model the force-defection relationships, and a pneumatic valve positioner application is demonstrated.
\end{abstract}

\section{Introduction}

Ortho-planar mechanisms are mechanisms with links that can be simultaneously located in a plane with motion out of that plane [1]. Compliant ortho-planar mechanisms gain their mobility from the deflection of flexible members. This paper discusses a new type of compliant ortho-planar mechanism - one with linear output motion along an axis orthogonal to the fabrication plane. These devices, called compliant ortho-planar springs, have force-deflection relationships that make them behave like springs, but they have several advantages over traditional springs.

1. Corresponding author. 
Examples of previously existing compliant or ortho-planar type springs are spider springs, geophone springs, volute springs, disc springs, and belleville springs. These types of springs are used in bolt assemblies, disc brake assemblies, valves, pneumatic controllers, and many other applications.

A major advantage of these types of springs is that they are very compact and, in many cases, they can be easily manufactured. In this paper a brief review of current ortho-planar springs will be followed by the description of a new family of compliant ortho-planar springs that provides significant advantages over current spring designs.

\section{Current Ortho-Planar Type Springs}

An ortho-planar spring can be defined as a spring which can be either fabricated in or compressed down into a single plane. Some of the springs that fit this definition are the disc spring (Figure 1a), volute (conical) springs (Figure 1b), and the spider (geophone) springs (Figure 1c). The disc spring is manufactured in a slightly out-of-plane position and provides resistance as it is forced down towards the plane. The volute spring is a thin strip of steel wound so that the coils fit inside of each other. It also provides resistance as it is forced down towards the plane. The spider spring is usually manufactured in the plane and provides resistance as it is forced in either direction out of its plane of fabrication. More discussion on currently existing springs is available in common sources such as [2], [3], and [4].

\section{Ortho-Planar Spring Advantages}

A major advantage of ortho-planar springs is that they are very compact. These springs are designed to take up as little room as possible in their compressed or uncompressed position. Another advantage is that they are easy to manufacture because they can be made from a single piece of material. 


\section{Disadvantages of Current Ortho-Planar Springs}

One of the disadvantages of a spring like the spider and volute springs is that they require some rotation to occur during their operation. Because of this rotation, anything fixed to the surface of the platform will be required to rotate with the platform. Also, if the platform is not fixed to the adjacent part then the two components are left to slide against each other, causing wear to both parts as well as vibration and noise. If the platform shape is not circular, a rotating platform may require more clearance in order to avoid contact with nearby structures. One final disadvantage is that rotation in the spider spring legs adds torsional stresses to the members, and reduces the total possible deflection and the fatigue life of the spring.

The disc springs, like the Belleville, require clearance for either the outer or inner edges of the spring to slide. The assembly tolerances are also required to be quite tight, increasing the cost of the assembly.

\section{A New Ortho-Planar Spring Design}

A new type of ortho-planar spring has been designed that has the potential of possessing all of the advantages of conventional ortho-planar springs without most of the disadvantages. This new spring operates by raising or lowering its platform relative to the base (see Figure 2) without any rotation of this platform in the $x, y$, or $z$ direction. Because of its planar nature it can be fabricated using any number of fabrication methods, including stamping, laser cutting, water jet cutting, wire EDM, milling, and injection molding. The fully compliant version requires no assembly and is very compact. It can be constructed out of many kinds of materials. Prototypes have been constructed of several materials including stainless steel, aluminum, polypropylene, and polycrystaline silicon. (Photographs of the prototypes can be found in [5]).

Two designs are presented in detail: the radial design and the side design. The radial leg design has its flexible segments extending radially away from the platform's center (Figure 2a). The side leg design has its flexible segments offset from the radial attachments (Figure 2b). Both leg designs result in non-rotational motion of the platform. 
The remainder of this section will establish some of the nomenclature for these new mechanisms. After that, a section is devoted to the radial-leg design followed by a section on the standard side-leg design. Once these have been presented, a brief discussion on performance of the ortho-planar springs will be displayed. The concluding section is devoted to additional configurations.

\section{Nomenclature}

The introduction of definitions and terms will be useful when discussing the ortho-planar designs that have been created. All of the designs that follow are made unique by variations on the three basic components of the design: the number of legs, the number of flexible segments in each leg, and the leg style or class. A single leg is defined as all of the segments between the base and the platform on a given side (i.e. the flexible segments and the intermediate platform). A three leg device is illustrated in Figure 3.

Within all of the classes of the newly designed springs, the number of legs that attach to the platform can be as few as 2 and as many as desired. For the purposes of naming the various designs, a classification system has been created which uses the number of legs as the first descriptive section in the name. The system uses the terms Bi, Tri, Quad, and Pent to represent the leg totals of two, three, four, and five respectively.

The second section of the name describes the number of flexible segments found between the base and the intermediate platform, and between the intermediate platform and the platform within each leg. These numbers are separated by a dash which represents the intermediate platform. The classification Tri 2-1 would indicate that the mechanism has three legs and that each leg has two flexible segments between the base and the intermediate platform, and one flexible segment between the intermediate platform and the platform. It is possible to have a different number or different arrangement of flexible segments on each leg, in which case each leg is called out individually and separated by a colon (e.g. Tri 2-1:1-1:1-2). This can be extended for devices with more legs.

The terms radial and side are used to describe separate classes of the new ortho-planar spring. While these are referred to as the two standard styles, other styles and variations will be presented 
later. For now it is important to say that the leg style is the third section in the newly established classification system. A radial-leg style is represented by the letter $\mathrm{R}$ and the side-leg style is represented by the letter S. Other letters and numbers that are found in this section of the mechanism name will be discussed as they are introduced.

To summarize, the three major components of the ortho-planar springs are used as the three sections of a classification system as seen here:

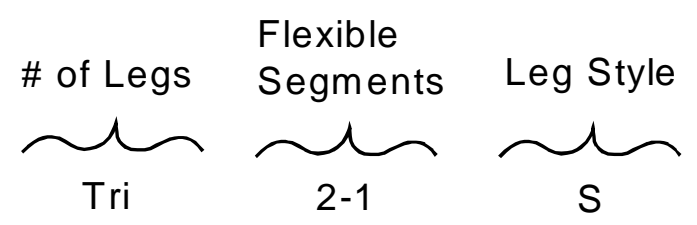

Table 1 shows how some of these names are formed.

\section{Pseudo-Rigid-Body Model}

When the deflections of the flexible segments are large enough to introduce geometric nonlinearities, linear beam equations are not adequate to accurately predict their behavior. The pseudorigid-body model has been developed to simplify the analysis of compliant mechanisms that undergo large nonlinear deflections. Flexible segments are modeled as rigid links with revolute joints and torsional springs located such that they accurately describe the motion and stiffness of the member. In this way a compliant mechanism can be converted to a rigid-body mechanism for analysis purposes.

Table 1: Examples of how ortho-planar spring names are formed using the new classification system.

\begin{tabular}{|l|l|l|l|}
\hline $\begin{array}{l}\text { Number } \\
\text { of Legs }\end{array}$ & \multicolumn{1}{|c|}{ Flexible Segments } & $\begin{array}{c}\text { Leg } \\
\text { Style }\end{array}$ & \multicolumn{1}{|c|}{ Name } \\
\hline Three & $\begin{array}{l}\text { 2 flexible segments between the base and } \\
\text { inter. platform and 1 flexible segment } \\
\text { between inter. platform and platform }\end{array}$ & Radial & Tri 2-1R \\
\hline Two & $\begin{array}{l}\text { One side has a 2-2 configuration and the other } \\
\text { has a 1-1 configuration. }\end{array}$ & Radial & Bi 2-2:1-1R \\
\hline Five & $\begin{array}{l}\text { 1 flexible segments between base and inter. } \\
\text { platform and 1 between inter. platform and } \\
\text { platform on all sides. }\end{array}$ & Side & Pent 1-1S \\
\hline
\end{tabular}


Pseudo-rigid-body models have been developed for various types of segments, including fixed-pinned ([6], [7]), functionally binary pinned-pinned [8], and others. The segment most critical for this work is for functionally binary fixed-guided segments, as shown in Figure 4. This type of segment is fixed at one end and the other end is constrained such that it does not rotate. The pseudo-rigidbody model for this segment is represented by a rigid link of length $r$ where

$$
r=\gamma L
$$

where $\gamma$ is the characteristic radius factor (usually $\gamma \approx 0.85$ ) [6], and $L$ is the length of the flexible segment. The torsional springs each have a torsional spring constant, $k$, of

$$
k=2 \gamma K_{\Theta} \frac{E I}{L}
$$

where $E$ is Young's modulus, $I$ is the area moment of inertia about the axis of bending, $K_{\Theta}$ is the stiffness coefficient (usually $K_{\Theta} \approx 2.65$ ) [7], and $L$ is the length of the flexible segment. The angle of the link is the pseudo-rigid-body angle, $\Theta$, and the torque at each torsional spring, $T$, is

$$
T=k \Theta
$$

where $\Theta$ is in radians. The coordinates of the end of the segment, $(a, b)$, are

$$
a=l(1-\gamma(1-\dot{\cos \Theta}))
$$

and

$$
b=\gamma l \sin \Theta
$$

The maximum stress in the segment, $\sigma_{\max }$, occurs at the wall and has a magnitude of

$$
\sigma_{\max }=\frac{P a c}{2 I}
$$


where $P$ is the applied force and $c$ is the distance from the neutral axis to the other fibers (usually half the thickness of the beam).

An alternative to the pseudo-rigid-body model in compliant mechanism design is the use of topology optimization. Examples of this approach can be found in [9] and [10].

\section{Radial Legs}

Within the radial-leg class, springs can be designed that contain any number of legs greater than 1 . Designs with 2, 3, 4, and 5 legs are briefly discussed followed by the introduction of a general set of equations for force displacement and stress.

\section{Bi Designs}

The simplest structure of the radial leg class is the two-leg style as illustrated in Figure 5. The platform of this mechanism does have the tendency to raise and lower straight up out of the plane without rotation in any direction. However, the pseudo-rigid-body model indicates that this mechanism is not limited to one degree of freedom. Figure 6 shows a side view of the two-leg design and its rigid-body equivalent.

The reason that this configuration displays more than one degree of freedom is because both legs allow for motion to occur along the same two axes. By creating the second leg at some position other than a 180 degree rotation from the first, motion can be allowed straight out of the plane $(z$ direction) while canceling out all side-to-side motion ( $x$ and $y$ ). However, doing this tends to decrease the stability of the platform. For these mechanisms, a stable platform is a platform that does not easily move out of its prescribed motion. The best way to limit the degrees of freedom while maintaining or increasing stability is to increase the number of legs.

\section{Tri Designs}

Increasing the number of legs to three increases the stability of the platform while decreasing the degrees of freedom to one. While the legs can be positioned at various angles around the platform, 
the most stable configuration is achieved when the legs are separated by 120 degrees. Successful prototyping of the tri designs has shown this to be an effective ortho-planar linear spring.

\section{Quad and Pent Designs}

Configurations of these ortho-planar springs containing both four and five legs were also designed and prototyped. Quad designs do not exhibit more stability than those designs of similar dimensions and three legs. Although, once again, the legs can be spaced at random angels, all of the designs prototyped applied a uniform 90 degree angular spacing between legs. However, this type of symmetric spacing can cause the platform to be somewhat unstable to rotations about its $x$ and $y$ axes.

The pent design is the most stable of all designs mentioned thus far (Figure 7). This design was also prototyped out of polypropylene. The added stability is partly due to the fact that adding more legs of the same parameters creates a higher overall stiffness. However, it is also more stable because of the positioning of the legs. If a uniform offset is used then each leg group is angled 72 degrees from its neighbor. This configuration reduces the instabilities that can occur by the twisting of the flexible segments about their long axes.

\section{Radial Leg Designs of Unequal Length}

Although the easiest and usually preferred spring designs contain equal length flexible segments, equal length is not required. In other words, as shown in Figure $8, \mathrm{~L}_{1}$ usually is equal to $\mathrm{L}_{2}$ and $\mathrm{L}_{1}$ is usually equal to $\mathrm{L}_{4}$. However, the required parameter for the defining motion is that the sum of the essential flexible segments be the same for each leg of the spring $\left(\mathrm{L}_{1}+\mathrm{L}_{2}=\mathrm{L}_{3}+\mathrm{L}_{4}=\mathrm{L}_{5}+\mathrm{L}_{6}=\right.$ $\mathrm{L}_{7}+\mathrm{L}_{8}$ ). While deviations from these requirements may produce close to linear non-rotational motion, the pseudo-rigid body model indicates that it is not a linear motion.

One of the disadvantages of unequal length-link designs is that the stresses will be higher in the shorter segments. Another disadvantage may be the inefficient use of space to achieve a specified motion. By shortening one of the segments the maximum possible displacement is reduced and the force required to reach any given distance is increased. 


\section{Side-leg Designs}

The designs shown thus far have had the flexible segments extending radially away from the platform. Another class of the ortho-planar springs has its flexible segments positioned to the side of the platform (Figure 9). The positioning of the flexible segments in this side-leg design produces a very compact variation of the spring. A comparison between leg positions will be discussed after a few characteristics of this side-leg design are presented.

Just as those designs with flexible segments extending radially away from the platform, the side-leg design produces multi-degree of freedom or unstable mechanisms with a two-leg design. Designs of three, four, and five legs, however, are more stable.

\section{Tri Designs}

With three legs positioned 120 degrees apart (Figure 2b), a stable one-degree-of-freedom mechanism is produced.

\section{Quad and Pent Designs}

Ortho-planar configurations of both the quad (Figure 9) and pent (Figure 10) designs were constructed out of polypropylene. As is the case with the radial leg designs, the tri designs are more stable than the quad designs. The quad designs appear especially unstable to rotations about the $x$ and $y$ axes.

The five-leg designs are more stable, and it is possible that devices with more legs will be even more stable. However, each individual leg tends to rotate about the long axis of its flexible segment. When a radial or side leg design is used, if the leg count is even, then each leg has a leg directly opposite it on the platform that has similar rotational tendencies. Thus, odd number leg counts are more stable than similar even leg count devices (Figure 11). 


\section{Rotation}

Unlike geophone (spider) springs, these ortho-planar springs do not experience significant rotation of the platform. Eliminating rotation eliminates the disadvantages associated with rotation discussed previously.

\section{Stress for a given deflection}

Stress for small deflections will be considered first, followed by a discussion of the stress associated with large deflections.

The general equation that holds true for both large and small deflections of bending stress is

$$
\sigma=\frac{M c}{I}
$$

where $M$ is the moment load, $c$ the distance from the neutral axis to the edge, and $I$ is the moment of inertia. The maximum stress is produced by the maximum moment which is described by

$$
M_{M A X}=\frac{F L}{2}
$$

where $F$ is the vertical force being applied and $L$ is the length of one flexible segment.

The displacement for a single small-deflection flexible member is a function of force [11]

$$
\delta=\frac{F L^{3}}{12 \cdot E I}
$$

and can be rearranged into

$$
F=\frac{12 \delta E I}{L^{3}}
$$

so that we can substitute equation (10) into (8) and then substitute this equation into equation (7). Because the displacement of the platform is twice that of the intermediate platform, the maximum stress is 


$$
\sigma_{M A X}=\frac{3 \cdot \delta_{\text {platform }} \cdot E \cdot c}{L^{2}}
$$

For large deflections we start with the same basic stress equation. This time, however, the moment and the force equations are more complicated. Using the pseudo-rigid-body model of one fixed-pinned flexible segment (Figure 12) results in the following stress equation [12]:

$$
\sigma_{M A X}=\frac{2 K_{\Theta} E c(1-\gamma(1-\cos \Theta)) \Theta}{L \cos \Theta}
$$

\section{Displacement for a Given Force}

In all of the fully compliant ortho-planar spring designs shown thus far, the displacement has been created by using long flexible fixed-guided segments. Although this is just one of several ways to produce the desired motion, it allows the ortho-planar springs to have large deflections and still be fully compliant (one-piece). These segments will therefore be the basis of the displacement equations discussed in this section.

The familiar spring equation of

$$
F=k \delta
$$

where $F$ is the force, $\delta$ is the displacement, and $k$ is the spring constant can be used to describe the force required for the displacement of one of the fixed-guided beams. If $n$ is defined to be the total number of flexible segments between the base and the intermediate platform, and $m$ is defined to be the total number of flexible segments between the intermediate platform and the platform, then

$$
\begin{aligned}
& n=s a \\
& m=s b
\end{aligned}
$$

where $s$ is the number of legs and $a$ is the number of segments between the base and the intermediate platform, and $b$ is the number of segments between the intermediate platform and the platform. For a 
given leg the springs between the base and intermediate platform may be considered to be springs in parallel and their spring constants are added together as

$$
F_{A}=\delta \sum_{i=1}^{a} k_{i}=\delta\left(k_{1}+k_{2}+\ldots+k_{a}\right)
$$

The same is the case for the springs between the intermediate platform and the platform, or

$$
F_{B}=\delta \sum_{i=1}^{b} k_{i}=\delta\left(k_{1}+k_{2}+\ldots+k_{b}\right)
$$

where $F_{A}$ is the force applied to the segments between the base and the intermediate platform and $F_{B}$ is the force applied to the segments between the intermediate platform and the platform. Defining

$$
k_{A}=k_{1}+k_{2}+k_{3}+\ldots+k_{n}
$$

and

$$
k_{B}=k_{1}+k_{2}+k_{3}+\ldots+k_{m}
$$

These equivalent springs are in series, which results in the equation

$$
\delta_{p}=\frac{F_{A}}{k_{A}}+\frac{F_{B}}{k_{B}}
$$

where $\delta_{p}$ is the displacement of the platform or $2 \delta$. But because

$$
F_{A}=F_{B}
$$

for a given leg, then

$$
\delta_{p}=\left(\frac{k_{A}+k_{B}}{k_{A} k_{B}}\right) F_{A}
$$


or

$$
F_{A}=\frac{k_{A} k_{B}}{k_{A}+k_{B}} \delta_{p}
$$

For all legs, the total force is

$$
F_{A}=\frac{s k_{A} k_{B}}{k_{A}+k_{B}} \delta_{p}
$$

If all of the flexible segments have the same value of $k$ the total equivalent spring will be

$$
k_{\text {total }}=\frac{s a k b k}{a k+b k}=\frac{s a b}{a+b} k
$$

or

$$
k_{\text {total }}=\frac{n m}{n+m} k
$$

When this is the case, the equivalent spring can be quickly calculated. For example, a Tri 2-1 would indicate that

$$
\begin{aligned}
& n=3 \cdot 2=6 \\
& m=3 \cdot 1=3
\end{aligned}
$$

For small deflections, the displacement of the deflecting members can be defined using equation (9). It can be seen that the spring constant for an individual flexible segment, $k$, is

$$
k=\frac{12 E I}{L^{3}}
$$

For large deflections

$$
F=\left(\frac{n m}{n+m}\right)\left(\frac{12 K_{\Theta} E I\left(\Theta-\Theta_{0}\right)}{L^{2} \cos \Theta}\right)
$$


The angle $\Theta$ is related to the deflection of the platform, $\delta_{p}$, by

$$
\delta_{p}=2 \delta L \sin \Theta
$$

For large deflections, if the deflection is symmetric such that the deflection between the base and intermediate platform is equal to the deflection between the intermediate platform and the platform, then

$$
F=\frac{4 s K_{\Theta} E I \Theta}{L^{2} \cos \Theta}
$$

where

$$
\Theta=\operatorname{asin} \frac{\delta_{p}}{2 \gamma L}
$$

The symmetric deflection occurs when $K_{A}=K_{B}$. This is usually the case because it balances the stresses and a larger deflection is obtained for the same size spring when this condition is maintained. However, if the condition is not maintained then the nonlinear equations become much more complicated, and it would be as easy to use nonlinear finite element analysis.

\section{Other Configurations}

There are many configurations of these new ortho-planar springs that have not yet been discussed. Configurations of 2, 3, 4, and 5 legs have been described, but other numbers of legs are possible. Any of these designs can be created in a fully compliant or partially compliant configuration. Besides these additional configurations, new configurations can be created by using the following: multiple-platforms, multiple flexible segments per leg, curved flexible segments, various angles of attachment, and inversions. 


\section{Multiple-Level Platforms}

Single level platforms can be combined together to produce multi-story mechanisms, like the one shown in Figure 13. The original platform becomes a subplatform that contains within it a second fully functioning spring. There is no theoretical limit to the number of expansions possible. It is also not a requirement that each level use either the same number of legs or the same type of legs as its predecessor. In continuing with the established classification system of these springs, a multi-level platform is named using the + symbol between complete ortho-planar springs (e.g. Quad 1-1S + Quad $1-1 S)$.

\section{Multiple Flexible Segments per Leg}

In all of the designs shown thus far, each leg of the design contained exactly two essential flexible segments per leg. Increasing the number of segments to three or more produces an alternative configuration of the new ortho-planar springs with additional intermediate platforms (Figure 14). Increasing the number of flexible segments per leg increases the potential displacement of the platform using the same length flexible segments. However, it can also decrease the stability of the platform, especially with four or more segments per leg. In keeping with the established nomenclature, these mechanisms simply have additional intermediate platforms and therefore only require additional dashes (e.g. Quad 2-2-1R).

\section{Curved Flexible Segments}

Although all of the flexible segments discussed up to this point have been straight when undeflected, curved beams are also acceptable (Figure 15). In some cases curved beams can be used to create an even more compact design than would be possible with straight beams. To continue with the established nomenclature, curved beams are indicated by an additional letter $\mathrm{C}$ in the flexible segment section of the name. This shape was also successfully prototyped. Other shapes for the flexible segments can also be used. 


\section{Various Leg Offsets or Angles of Attachment}

One way to look at the difference between the side and radial leg designs is to view the side design as a radial design with offset legs (Figure 16a). Using a circular platform, it is feasible to attach the legs at the standard offset positions (radial and side designs) or any other amount of offset in-between the standard side and radial designs (Figure 16b).

However, when using a non-circular platform it may be easier to compare these differences by looking at the angle formed between the line orthogonal to the platform and the first flexible leg. Under this system the radial leg becomes the zero degree design and the side leg the 90 degree design. It is not only possible to create various angles of attachment between 0 and 90 degrees, but also angles greater than 90 degrees (Figure 17). Some of these greater than 90 degree attachments may require long attachment bars that extend away from the platform. If this method is used, an extension to the leg class can be added to indicate the angle of attachment (e.g. Tri 1-1S 45).

\section{Inversions}

An inversion is created by choosing a different link to be ground [13]. Inversions of these newly designed ortho-planar mechanisms are created by fixing all of the intermediate platforms or fixing the platform. Fixing the intermediate platforms can cause the creation of a structure with zero degrees of freedom or it can increase the number of degrees of freedom, depending on the arrangement of the flexible segments. Fixing the platform results in the same basic ortho-planar spring where the platform becomes the base and the base becomes the platform.

\section{Example Application}

An orthoplanar spring was developed for use in a pneumatic valve controller as shown in Figure 18, and was laser cut from 0.01 inch thick stainless steel. A ferrous component was attached to the center of the spring and it was then placed near an outlet nozzle as shown in Figure 19. When an electric current goes through the coil it creates a magnetic field. This magnetic field pulls the spring toward the nozzle, which in turn restricts the flow of air through the nozzle. Restricting the flow 
causes the pressure to increase and the diaphragm deflects, moving the spool valve. The spool valve controls the pressure that positions a much larger pneumatic valve. In this way a small current can be used to position a large valve. This device was successfully implemented in an industrial valve for an international valve manufacturer (Flowserve). Advantages of the orthoplanar spring include its compactness, ease of manufacture (it can be stamped), and its parallel motion which makes it easy to calibrate and less sensitive to variation in the assembly.

\section{Conclusions}

The compliant ortho-planar spring introduced in this paper is compact and can undergo a large displacement in either direction. It has advantages over spider springs in that the platform does not rotate in its motion, does not introduce torsional stresses in the flexible segments, and can undergo larger deflections for a given size. It has advantages over disc-type springs in that it is does not require sliding motion between parts connecting to the spring, can undergo larger displacements, and it not as sensitive to variation in assemblies. The lack of rotation and sliding means that the ortho-planar spring can be directly attached to adjacent parts without relative motion. This reduces wear, noise, and reduces particulates caused by abrasive motion in rubbing parts. The particulate reduction can be particularly important in sensitive environments such as microelectronic fabrication equipment.

Springs with multiple legs can be designed, but an odd number of legs was found to be most beneficial. The legs can be at any angle but the radial and side leg designs are important special cases that are described in detail. The nomenclature introduced in this paper is valuable for easily identifying parts of the ortho-planar spring and the configuration.

\section{Acknowledgments}

This research was supported by a National Science Foundation CAREER Award under grant No. DMI-9624574. The assistance of Jason Briscoe, Nathan Burnside, Troy Gomm, Curtis Huffmire, John Larsen, Scott Thomson, and Shae Lorenc (the Flowcon capstone team) in designing, prototyping, and testing the spring for the valve controller is gratefully acknowledged, as is the financial and 
technical assistance of Flowserve. The authors also appreciate the work of Brent Weight in fabricating prototypes of other configurations, and for Eric Sipe in his assistance in compiling this paper.

\section{References}

[1] Parise, J.J., Howell, L.L., and Magleby, S.P., 2000, “Ortho-Planar Mechanisms,” Proceedings of the 2000 ASME Design Engineering Technical Conferences, DETC2000/MECH-14193.

[2] Wahl, A.M., 1963, Mechanical Springs, McGraw-Hill.

[3] Juvinall, R.C., 1983, Fundamentals of Machine Component Design, John Wiley \& Sons.

[4] Parmley, R.O., Editor, 1985, Mechanical Components Handbook, McGraw-Hill.

[5] Parise, J.J., 1999, “Ortho-Planar Mechanisms,” M.S. Thesis, Brigham Young University, Provo, Utah.

[6] Howell, L.L., Compliant Mechanisms, John Wiley \& Sons, New York, New York.

[7] Howell, L.L., Midha, A., and Norton, T.W., 1996, "Evaluation of Equivalent Spring Stiffness for Use in a Pseudo-Rigid-Body Model of Large-Deflection Compliant Mechanisms," Journal of Mechanical Design, Trans. ASME, Vol. 118, pp. 126-131, March, 1996.

[8] Edwards, B.T., Jensen, B.D., and Howell, L.L., 1999, "A Pseudo-Rigid-Body Model for Functionally Binary Pinned-Pinned Segments Used in Compliant Mechanisms," Proceedings of the 1999 Design Engineering Technical Conferences, DECT99/DAC-8644.

[9] Pedersen, C.B., Buhl, T., and Sigmund, O., 1999, “Topology Synthesis of Large-Displacement Compliant Mechanisms," Proceedings of the 1999 Design Engineering Technical Conferences, DETC99/DAC-8554.

[10] Saxena, A., and Ananthasuresh, G.K., 1999, "Towards the Design of Compliant Continuum Topologies With Geometric Nonlinearity," Proceedings of the 1999 Design Engineering Technical Conferences, DETC99/DAC-8578.

[11] Young, W.C., 1989, Roark's Formulas for Stress and Strain, Sixth Edition, McGraw-Hill.

[12] Derderian, J., 1996, "The Pseudo-Rigid-Body-Model Concept and Its Application to Micro Compliant Mechanisms," M.S. Thesis, Brigham Young University, Provo, Utah.

[13] Erdman, A.G., and Sandor, G.N., 1997, Mechanical Design: Analysis and Synthesis, Third Edition, Prentice Hall. 


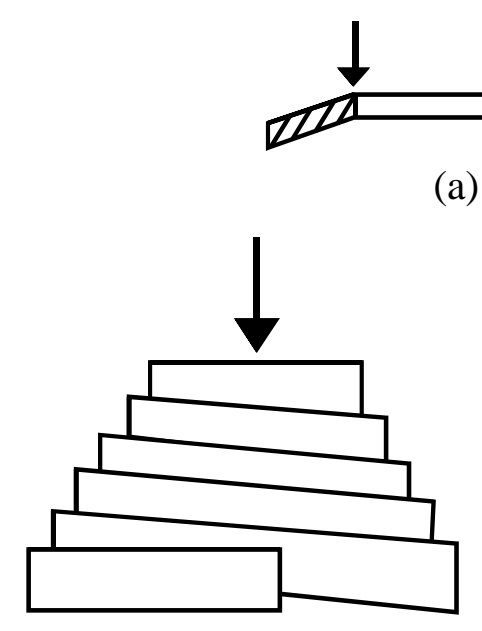

(b)

(a)

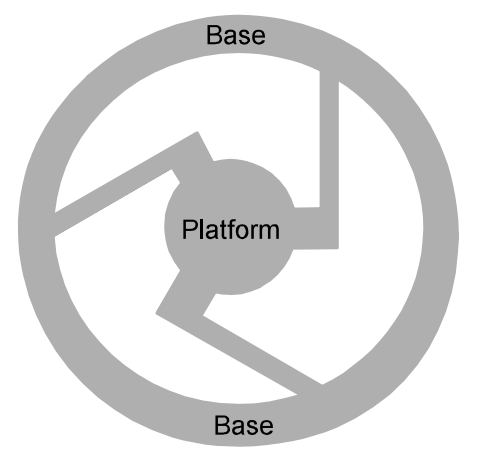

(c)

Figure 1 Examples of ortho-planar springs. (a) The cross-section of a Belleville disc spring. (b) The side view of a volute spring. (c) The top view of a spider spring.

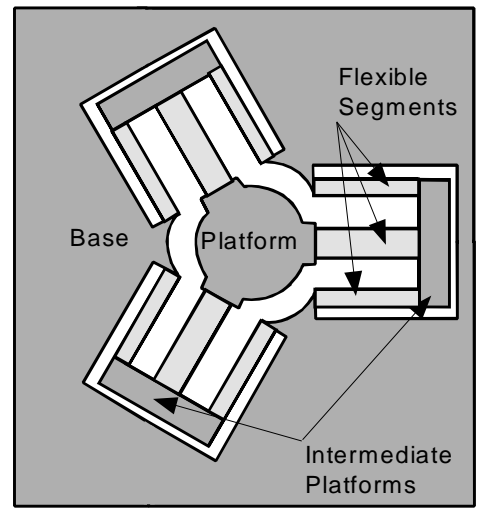

(a)

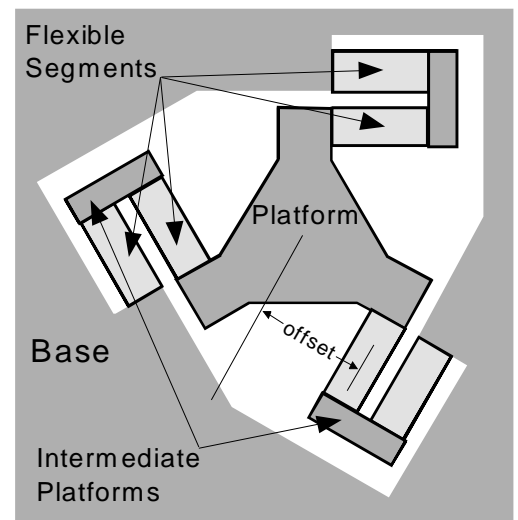

(b)

Figure 2 The new compliant ortho-planar spring. (a) An example radial design, Tri 2-1-R. (b) An example side design, Tri 1-1S. 


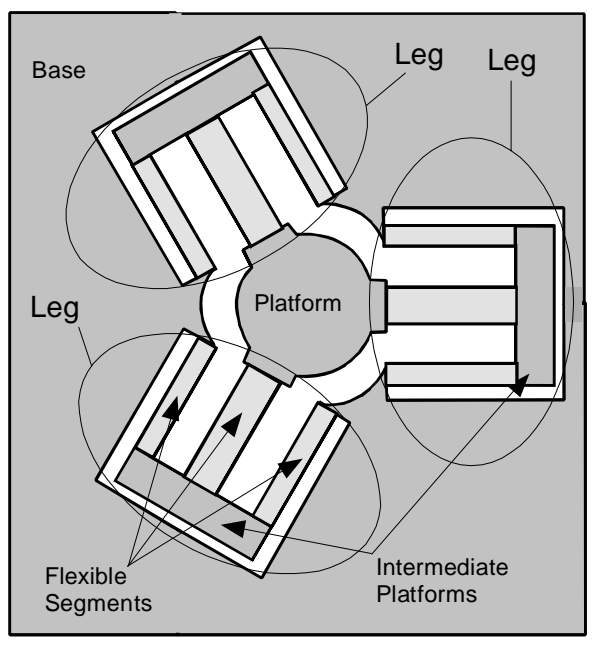

Figure 3 The circled areas indicate each of the three legs in this design, the Tri 2-1R.

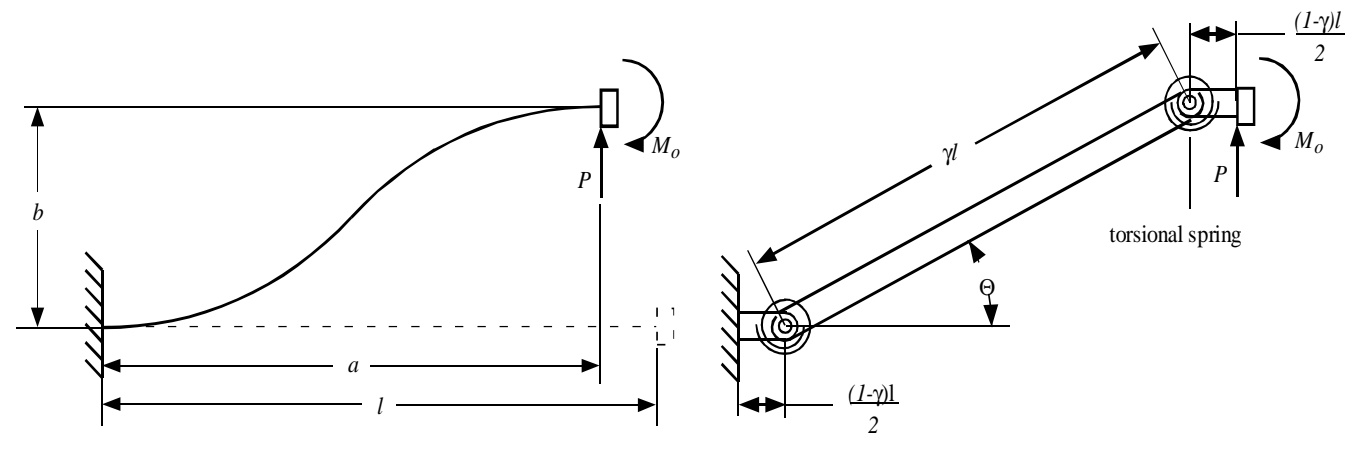

Figure 4 Fixed guided segment and its pseudo-rigid-body model.

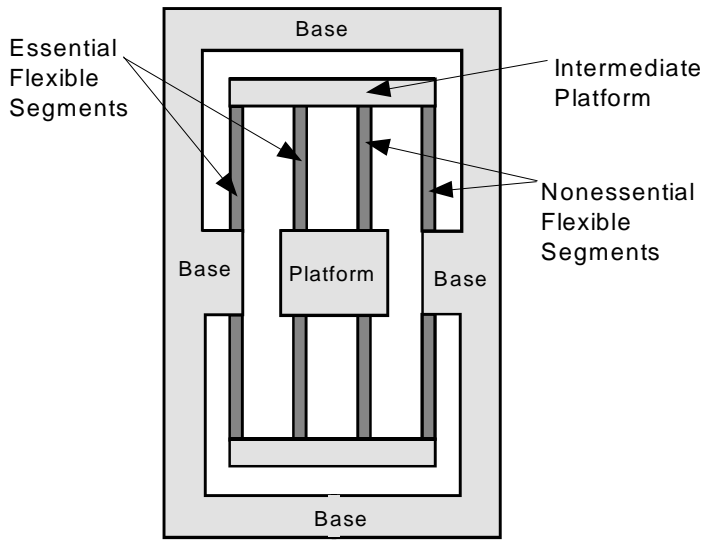

Figure 5 Plan view of a Bi 2-2R ortho-planar spring. Notice that only two flexible segments in series are required between the base and the platform. 

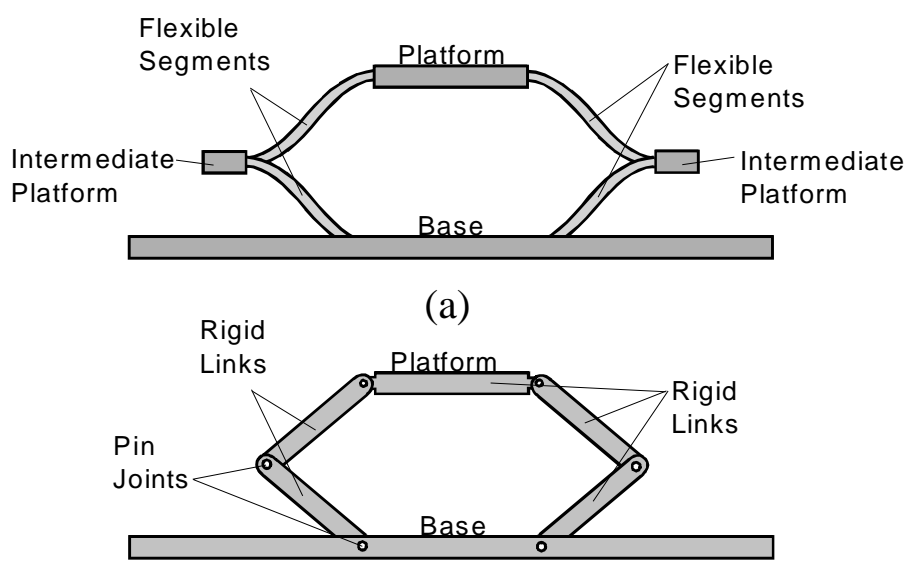

(b)

Figure 6 Side-view of the (a) Bi 1-1R design and (b) its rigid body equivalent or pseudo-rigid body model excluding torsional springs.

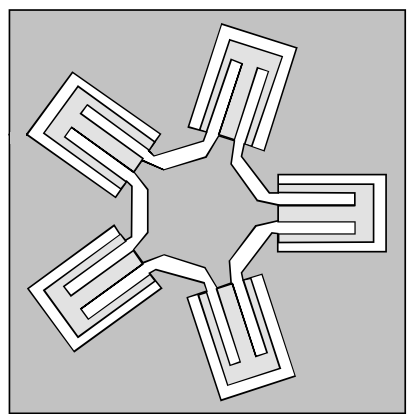

Figure 7 Plan view of the Pent 2-1R. $\quad$ Figure 8 Quad 2-1R design with unequal length flexible segments. $\mathrm{L}_{1}+\mathrm{L}_{2}=\mathrm{L}_{3}+\mathrm{L}_{4}=\mathrm{L}_{5}+\mathrm{L}_{6}=\mathrm{L}_{7}+\mathrm{L}_{8}$. 


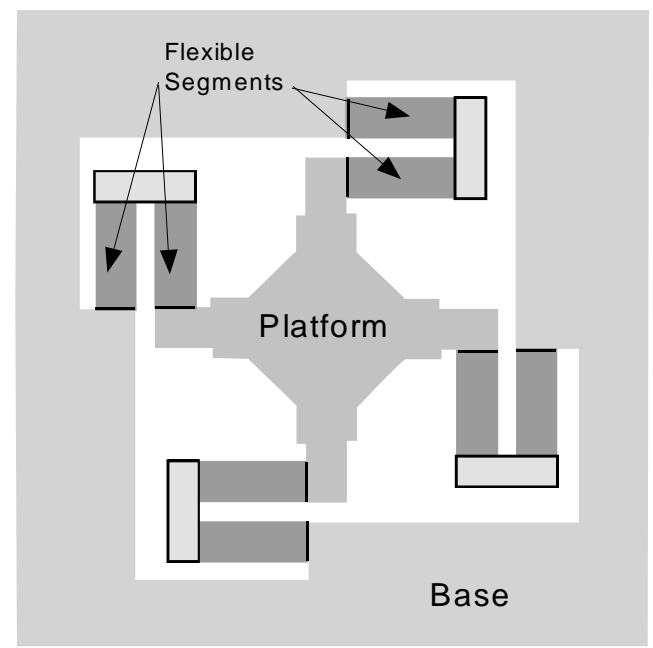

Figure 9 A Quad 1-1S design of the new ortho-planar springs.

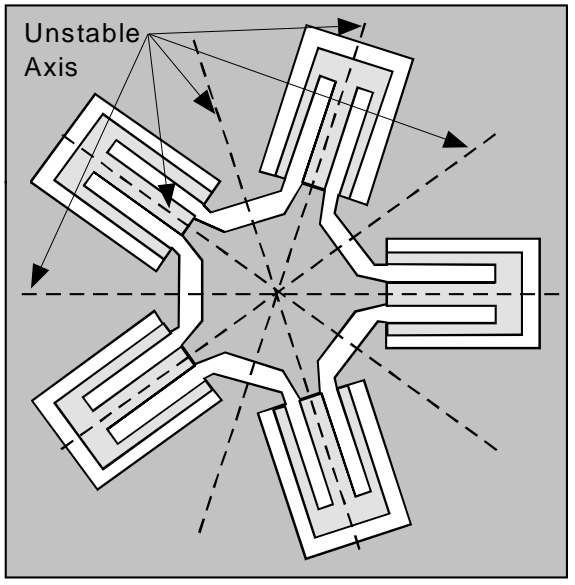

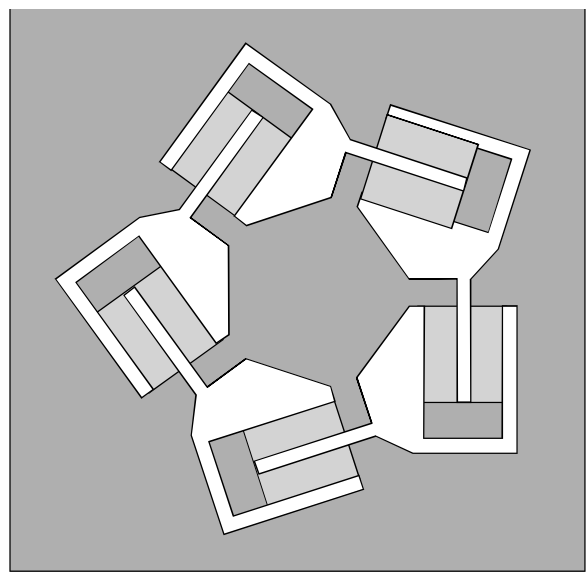

Figure 10 A Pent1-1S design.

Figure 11 The unstable axes of the (a) Pent2-1R and the (b) Hex2-lik.

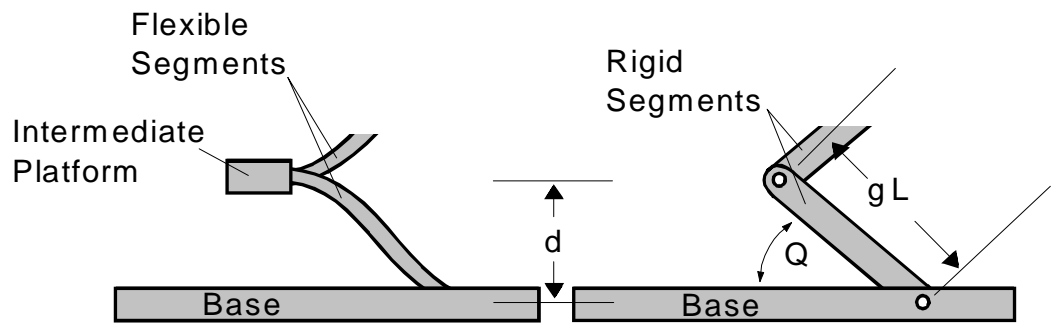

Figure 12 Part of a fully compliant ortho-planar spring and its pseudo-rigid-body model. 


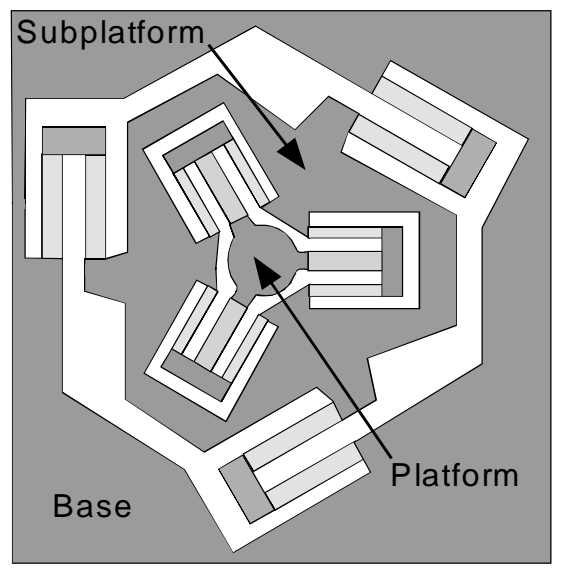

Figure 13 Plan view of a multi-level platform, Tri 1-1S+Tri 2-1R.

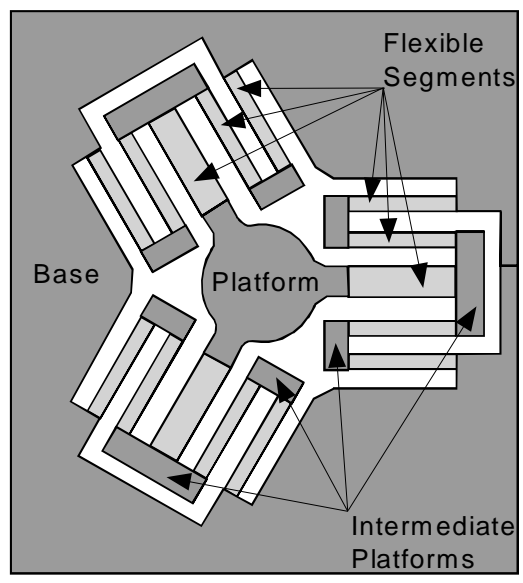

Figure 14 Plan view of a Tri 2-2-1R.

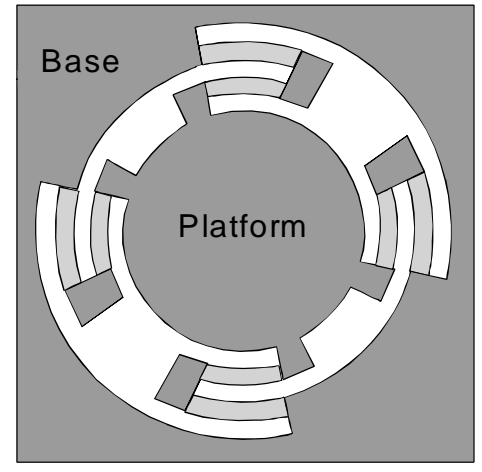

Figure 15 Plan view of a quad 1-1SC.

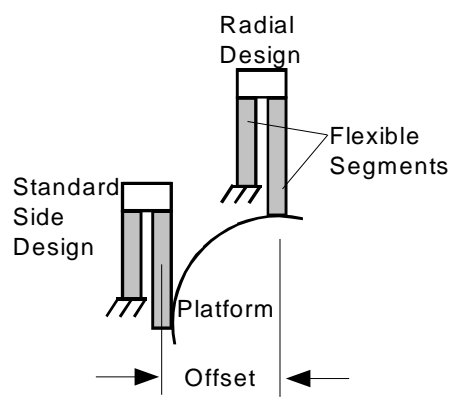

(a)

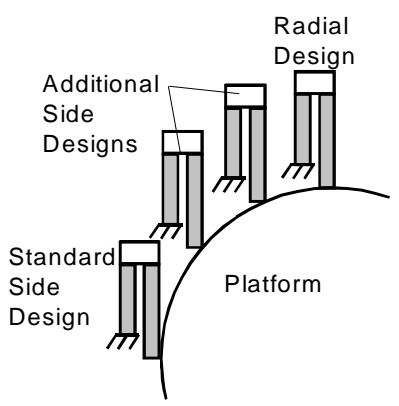

(b)

Figure 16 Changing the leg offsets can create (a) the radial and side designs as well as (b) additional designs. 


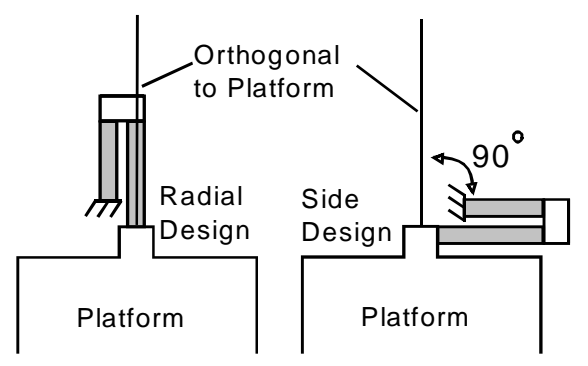

(a) (b)

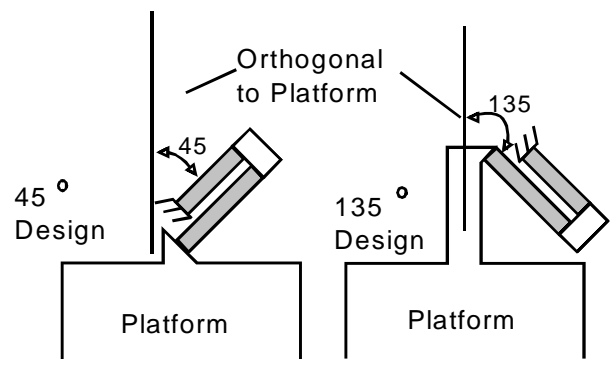

(c)

(d)

Figure 17 Various angles of attachment. (a)Radial or zero degrees, (b) side or 90 degrees, (c) 45 degrees, and (d) 135 degrees.

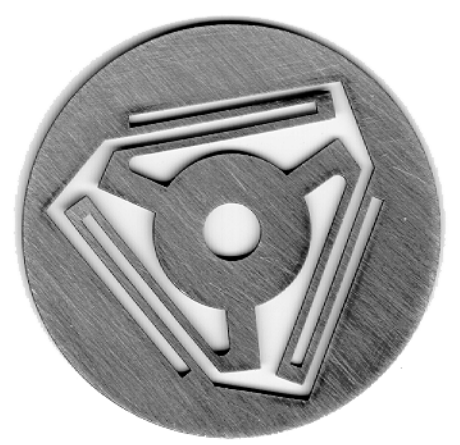

Figure 18 Prototype for use in a pneumatic valve controller for Flowserve Inc. This prototype was created from 0.01 inch stainless steel and measures approximately 1.5 inches in diameter.

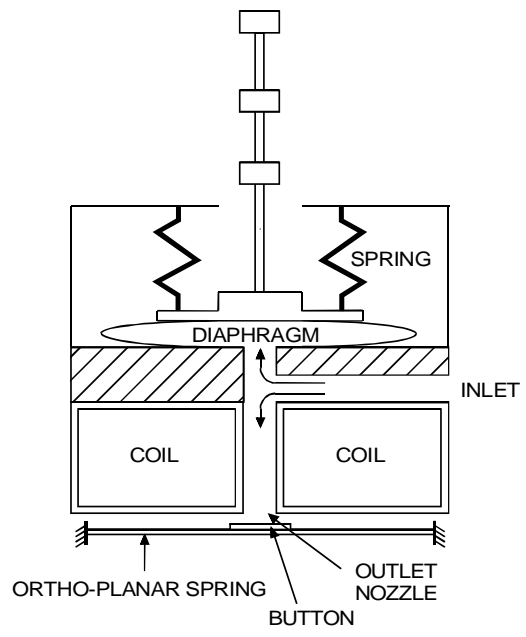

Figure 19 Orthoplanar spring application in industrial valve for Flowserve. 


\section{Figure Captions}

Figure 1 Examples of ortho-planar springs. (a) The cross-section of a Belleville disc spring. (b) The side view of a volute spring. (c) The top view of a spider spring.

Figure 2 The new compliant ortho-planar spring. (a) An example radial design, Tri 2-1-R. (b) An example side design, Tri 1-1S.

Figure 3 The circled areas indicate each of the three legs in this design, the Tri 2-1R.

Figure 4 Fixed guided segment and its pseudo-rigid-body model.

Figure 5 Plan view of a Bi 2-2R ortho-planar spring. Notice that only two flexible segments in series are required between the base and the platform.

Figure 6 Side-view of the (a) Bi 1-1R design and (b) its rigid body equivalent or pseudo-rigid body model excluding torsional springs.

Figure 7 Plan view of the Pent 2-1R.

Figure 8 Quad 2-1R design with unequal length flexible segments. L1 + L2 = L3 + L4 = L5 + L6 = L7 + L8.

Figure 9 A Quad 1-1S design of the new ortho-planar springs.

Figure 10 A Pent1-1S design.

Figure 11 The unstable axes of the (a) Pent2-1R and the (b) Hex2-1R.

Figure 12 Part of a fully compliant ortho-planar spring and its pseudo-rigid-body model.

Figure 13 Plan view of a multi-level platform, Tri 1-1S+Tri 2-1R.

Figure 14 Plan view of a Tri 2-2-1R.

Figure 15 Plan view of a quad 1-1SC.

Figure 16 Changing the leg offsets can create (a) the radial and side designs as well as (b) additional designs.

Figure 17 Various angles of attachment. (a)Radial or zero degrees, (b) side or 90 degrees, (c) 45 degrees, and (d) 135 degrees.

Figure 18 Prototype for use in a pneumatic valve controller for Flowserve Inc. This prototype was created from 0.01 inch stainless steel and measures approximately 1.5 inches in diameter.

Figure 19 Orthoplanar spring application in industrial valve for Flowserve. 
